\title{
Face Image Set Recognition Based on Improved HOG-NMF and Convolutional Neural Networks
}

\author{
Lixiu Hao*, Weiwei Yu \\ College of Information Engineering, Shanghai Maritime University, Shanghai 201306, China
}

\begin{abstract}
Objective Face recognition can be affected by unfavorable factors such as illumination, posture and expression, but the face image set is a collection of people's various angles, different illuminations and even different expressions, which can effectively reduce these adverse effects and get higher face recognition rate. In order to make the face image set have higher recognition rate, a new method of combining face image set recognition is proposed, which combines an improved Histogram of Oriented Gradient (HOG) feature and Convolutional Neural Network (CNN). Method The method firstly segments the face images to be identified and performs HOG to extract features of the segmented images. Secondly, calculate the information entropy contained in each block as a weight coefficient of each block to form a new HOG features, and non-negative matrix factorization (NMF) is applied to reduce HOG features. Then the reduced-dimensional HOG features are modeled as image sets which keep your face details as much as possible. Finally, the modeled image sets are classified by using a convolutional neural network. Result The experimental results show that compared with the simple CNN method and the HOG-CNN method, the recognition rate of the method on the CMU PIE face set is increased by about $4 \% \sim 10 \%$. Conclusion The method proposed in this paper has more details of the face, overcomes the adverse effects, and improves the accuracy.
\end{abstract}

Keywords: HOG; Non-negative matrix factorization; Convolutional Neural Network; Face image set recognition; Feature extraction

\section{Chinese Library Classification Number TP389.1}

\section{Introduction}

In the past ten years, methods based on convolutional neural network have made great progress in the field of computer vision, including face recognition, target recognition, action recognition and so on. In these fields, the progress of face recognition is particularly remarkable due to the development of two important aspects-intensive feature extraction and better loss function. In the field of face recognition, face recognition embedded by deep convolution network is considered as the most advanced method of face verification, face clustering and recognition. In reference $^{[1]}$, He Chun summarized that the recent research on face recognition is still devoted to improving the recognition rate of face.

The research of face image set recognition has attracted increasing attention in the field of computer vision. The difference between image set research and previous face recognition lies in that the previous face recognition inputs a single face image to recognize and extract the features of a single face, while the face image set consists in taking multiple images of a person as inputs, and the multiple images include changes of a person's posture, illumination, etc. Compared with a single image, the image set can describe individuals more effectively, which is easy to eliminate adverse effects and achieve the effect of improving recognition rate. In recent years, there are many methods for image set recognition and classification. Dong Xiwei et al. proposed a multi-manifold discriminant learning algorithm, which

\footnotetext{
Copyright (C) 2019 Lixiu Hao et al.

doi: 10.18063/phci.v2i1.1103

This is an open-access article distributed under the terms of the Creative Commons Attribution Non-Commercial License

(http://creativecommons.org/licenses/by-nc/4.0/), which permits unrestricted non-commercial use, distribution, and reproduction in any medium, provided the original work is properly cited.
} 
can accurately predict the intra-class changes in test samples ${ }^{[2]}$. Zhang Wenkai et al. proposed a multi-feature map fusion generation countermeasure network (MFF-GAN) model to complete image set identification. The image features extracted through multi-feature fusion can represent image details and high-level semantic information ${ }^{[3]}$. Ren Zhenwen et al. proposed an image set classification algorithm (SRNPC) based on entropy self-weighting joint regularization nearest point, which makes everyone classify more accurately and has better robustness ${ }^{[4]}$. Histogram of Oriented Gradients (HOG) is a feature extraction algorithm proposed by French researcher Dalal at the 2005 CVPR conference. It is used in pedestrian detection in conjunction with SVM classifier ${ }^{[5]}$. Xie and Sujata et al. used LBP and HOG algorithm to recognize faces from different angles, showing good results in occlusion and pose changes ${ }^{[6-8]}$. Sun Yue $e t$ al. used the combination of HOG and NMF to detect fast pedestrians. The algorithm significantly reduced the feature dimension and significantly improved the detection rate of linear support vector machine with the same computational complexity ${ }^{[9]}$. Lin Kezheng et al. proposed an information entropy weighted HOG feature extraction algorithm to avoid adverse effects such as illumination, which was also proved that the method has improved face recognition ${ }^{[10]}$. In the related research of image sets, feature extraction is rarely taken as an important step in face image set recognition. Feature extraction is very important in both individual face recognition and image set recognition. In the feature extraction part, the influence of local information on recognition effect is considered, which is helpful to improve face image and recognition. In this paper, a recognition method based on the combination of improved HOG-NMF features and convolutional neural network is proposed. The convolutional neural network can learn the local facial features, and the method has good robustness to the changes of facial pose and illumination. Experimental results show that this method improves the face recognition rate to a certain extent.

\section{Face Image Set Recognition}

Different from single face recognition, face image set does not provide a single image, but provides a set of images of the same object. Such an image set contains varied face images.

In the face recognition process of image sets, it is necessary to extract the features of each image. An image set represents an individual, then the features of an image set are composed of the features of all the images in its set and also represent the features of the individual. Information entropy weighted HOG feature extraction proposed by Lin Kezheng et al. can effectively avoid the adverse effects of illumination and posture, and is conducive to improving the overall recognition rate ${ }^{[10]}$. Modeling image sets is the focus of face recognition methods based on image sets, and it is also the key in recognition process. After face images set to be tested and the face image set in the database being both modeled, the modeled data is used as the input of the convolutional neural network, then the Softmax supervision function proposed by Wang Haibo et al. is used for category supervision, and finally the classifier is used for classification $^{[11]}$.

See Figure 1 for the general process of face recognition and Figure $\mathbf{2}$ for the process of face image set recognition: 


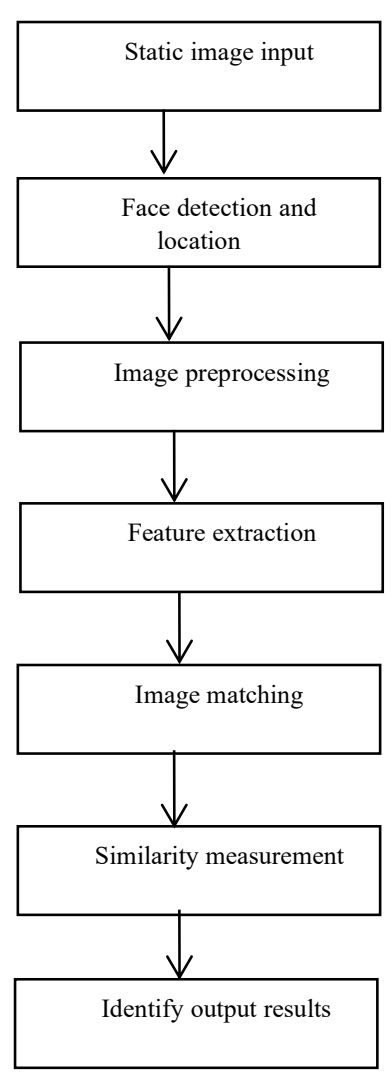

Figure 1. Basic process of face recognition

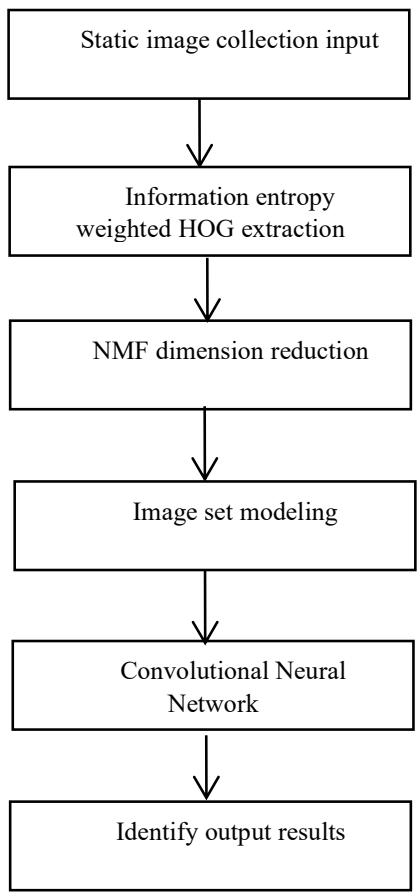

Figure 2. The face image set recognition process used in this paper

\section{Weighted HOG Feature Extraction}

Weighted HOG Feature Extraction: firstly, the face image to be recognized is divided into blocks, and the segmented image is subjected to HOG feature extraction. Then, the information entropy contained in each image is calculated as a weight coefficient and added to each block to form new HOG features. Finally, the features are reduced in dimension by NMF algorithm to obtain weighted HOG features. The algorithm steps are as follows:

(1) The gray scale of the image is transformed.

(2) The input image is standardized (normalized) in color space by Gamma correction method. The aim is to adjust the contrast of the image, reduce the influence of shadow and illumination changes on the local part of the image, and reduce image noise at the same time. In the texture intensity of the image, local surface exposure contributes a large proportion, so this compression process can effectively reduce the influence of shadow and illumination changes on the image ${ }^{[12]}$. The compression formula is as follows:

$I(x, y)=I(x, y)^{\text {gamma }}$

Where $I(x, y)$ represents the gray scale of pixel points $(x, y)$ in the image, gamma represents the correction coefficient of Gamma, and generally gamma takes 2.2 .

(3) Calculate the gradient (including size and direction) of each pixel of the image so as to capture contour information and further weaken the interference of illumination. The gradient of pixel points in the image is:

$G_{x}(x, y)=H(x+1, y)-H(x-1, y)$

$G_{y}(x, y)=H(x, y+1)-H(x, y-1)$

In the above formula, $g_{x}(x, y)$ and $g_{y}(x, y)$ respectively represent the horizontal gradient and vertical gradient at the pixel point $(x, y)$ in the input image, then the gradient amplitude and gradient direction at the pixel point $(x, y)$ are :

$G(x, y)=\sqrt{G_{x}(x, y)^{2}+G_{y}(x, y)^{2}}$ 
$\alpha(x, y)=\tan ^{-1}\left(\frac{G_{y}(x, y)}{G_{x}(x, y)}\right)$

(4) Divide the image into small cells (e.g. $3 * 3$ pixels /cell) to count the gradient histogram of each cell (the number of different gradients), and construct a gradient direction histogram descriptor for each cell. In this paper, the gradient direction of cell is divided into 9 direction blocks from 360 degrees.

(5) Each unit is formed into a block (e.g. $3 * 3$ cells/block), and HOG characteristics of the block are obtained by connecting the gradient direction histogram descriptor of all units in a block in series.

(6) Calculate the weight coefficient. When the face has different expressions, only a few key parts will change. Therefore, for each sub-block of the face image, different weight coefficients should be given. Let the image be divided into $\mathrm{m}$ sub-blocks, and the weight coefficient of the sub-block I is expressed as:

$E_{i}=-\sum_{k=0}^{\mathrm{n}} P_{i}^{k} \lg P_{i}^{k}$

In the above formula, $n$ represents the pixel gray level, i.e. $n=255, P_{i}{ }^{k}$ represents the probability of occurrence of the $k$-th pixel point, $\lg P_{i}^{k}$ represents the amount of information contained in the $k$-th pixel point, and the greater the information entropy of the sub-block, the greater the weight given to it, and the weight coefficient of the $i$-th sub-block is:

$W_{i}=\frac{E_{i}}{\sum_{i=1}^{m} E_{i}}$

The weighted gradient direction histogram formula for the $i$-th sub-block is:

$\mathrm{D}_{\mathrm{i}}=W_{i} *$ descriptor ${ }_{i}$

In the above formula, descriptor $i$ represents the HOG feature of the $i$-th sub-block, and $D_{i}$ represents the weighted HOG feature of the $i$-th sub-block.

The weighted HOG features $D_{i}$ of all block in the image are connected in series to form the HOG features of the image. The results of HOG feature extraction are shown in Figure 3. The HOG feature will be subjected to non-negative matrix dimensionality reduction (NMF) later.

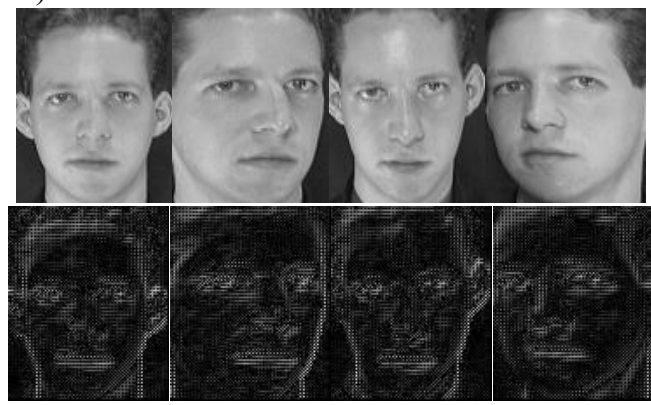

Figure 3. Image set after HOG feature extraction ((top)Original image; (bottom)Result image)

\section{Non-negative Matrix Factorization Algorithm (NMF)}

The non-negative matrix factorization (NMF) algorithm was originally a breakthrough of two scientists, D.D.Lee and H.S.Seung, in the study of non-negative matrices in mathematics (D.D.Lee et al., 2010). NMF matrix decomposition method decomposes the matrix into a matrix with only positive numbers, because negative numbers are meaningless in images. This decomposition method of matrix with only positive numbers is very meaningful for image recognition. The idea of non-negative matrix is to divide a matrix into two smaller matrices and multiply them. The matrix decomposition formula is as follows:

$V_{i u} \approx(W H)_{i u}=\sum_{a=1}^{r} W_{i a} H_{a \mu}$ 
$W_{i a} \leftarrow W_{i a} \sum_{\mu} \frac{V_{i \mu}}{(W H)_{i \mu}} H_{a \mu}$

$W_{i a} \leftarrow \frac{W_{i a}}{\sum_{j} W_{j a}}$

$H_{a \mu} \leftarrow H_{a \mu} \sum W_{i a} \frac{V_{i \mu}}{(W H)_{i \mu}}$

In the formula, $i$ and $u$ represent the size of the original matrix, $a$ represents the rank of the decomposed matrix, $r$ is the rank of the original matrix, $V$ is an approximation of the original matrix, and $W$ and $H$ are the two decomposed matrices. $W$ and $H$ are iterative algorithms, and the initial $W$ and $H$ are randomly selected.

An example of the operation results using this method is shown Figure 4:

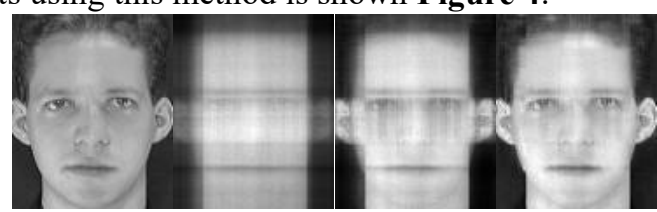

Figure 4. Effect of NMF on image dimensionality reduction

The first picture on the left of Figure 4 is the original picture, the second picture is the effect picture after 10 iterations, followed by the effect picture after 20, 40 and 100 iterations. It can be seen from the effect picture that the more iterations the picture is clearer, and the effect picture after 100 iterations is even clearer than the original one.

\section{Image Set Modeling}

An image set represents a target (person), and there are many images in the image set, which cover many changes of the person's face, such as posture, expression difference, light and shade, etc. Therefore, image set modeling needs to express these changes and describe the details. However, this paper is aimed at the recognition of image sets. Due to the influence of light, posture and expression on human face, the internal data of image sets no longer has linear features, which has an impact on analysis and recognition. In order to facilitate CNN recognition, this paper models the data set as an image set with linear features, that is, a person's image set is formed into an image set sample according to $\mathrm{n}$ images, common features are extracted from each image set sample, and then $\mathrm{CNN}$ is used for classification and recognition.

In this paper, in the process of image set modeling, it is assumed that a person's n pictures are modeled, and the arithmetic average value is calculated for each person's NMF dimension-reduced image pixel value, with the formula as follows:

$I(x, y)=\frac{\sum_{i=1}^{n} I_{i}(x, y)}{n}$

In the formula, $I_{i}(x, y)$ represents the HOG eigenvalue of $(x, y)$ of the $i$-th image of a person, $I(x, y)$ represents the HOG eigenvalue at $(x, y)$ of the image set after modeling of $n$ images, and $n$ represents the number of images in the image set. Define a person's image set, $X=\left\{x_{1}, x_{2}, x_{3}, \cdots, x_{N}\right\} \quad x_{i} \in R^{D}, \mathrm{R}^{\mathrm{D}}$ is a D-dimensional vector space, $N$ is the number of samples in the set. After modeling, the image set can be rewritten as $X=\left\{x_{11}, x_{12}, x_{13}, \cdots, x_{[\underline{\underline{N}}}\right\}$, the number of image sets in set $X$ takes the value $\left[\frac{N}{n}\lceil\cdot x 11\right.$ represents image set 1 after image set 1 is modeled. The linear modeling of the
image set is shown in Figure 5. 


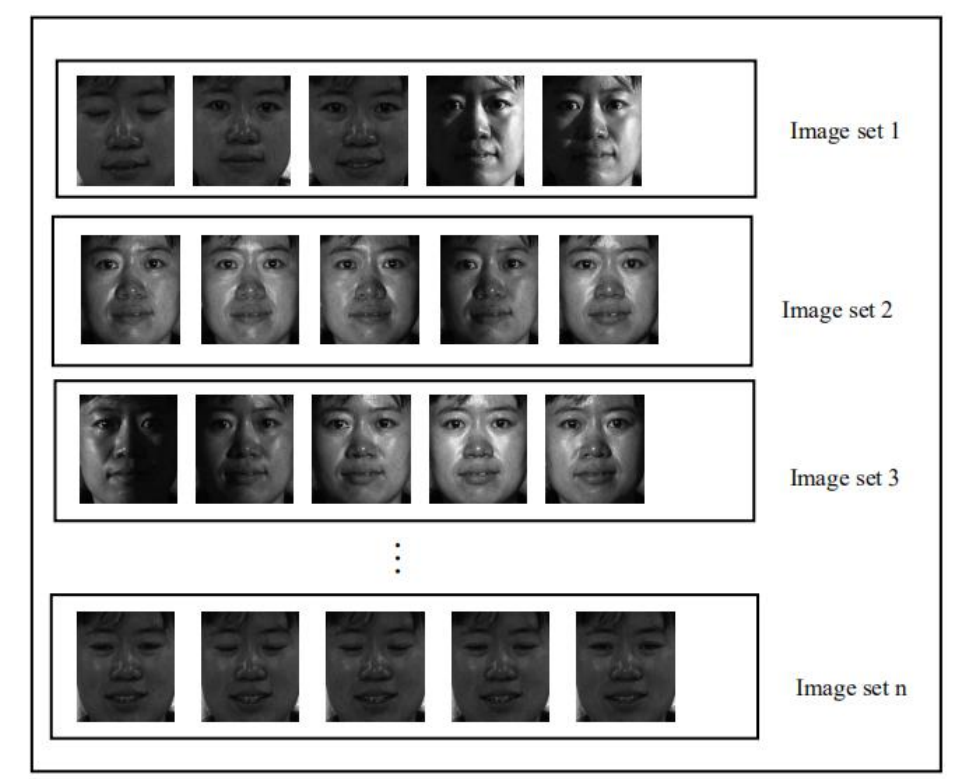

Figure 5. Image set modeling

\section{Convolutional Neural Network}

In the traditional fully connected neural network, every neuron between every two adjacent layers is connected with edges. When the feature dimension of the input layer is very large, the parameters of full connection network training will increase, which will affect the calculation speed. For example, in a black and white $28 \times 28$ picture, there are 784 neurons in the input layer. If only one hidden layer is used in the middle, and the hidden $\omega$ layer contains 15 neurons, there are $784 \times 15=11760$ parameters. If $28 \times 28$ RGB pictures are input, there are $28 \times 28 \times 3=2352$ input neurons. It is easy to see that too many training parameters are easy to occur in the process of image processing using fully connected neural networks.

However, in the Convolutional Neural Network (CNN), neurons in the convolutional layer are only connected to some neuron nodes in the previous layer, not all connected, and some neurons in the same layer share weights $\omega$ and offsets $b$, which will greatly reduce the number of training parameters. The structure of convolution neural network CNN generally includes input layer, convolution layer, excitation layer, pooling layer, full connection layer and output layer.

The structural design of convolution neural network in this paper is shown in Figure 6:

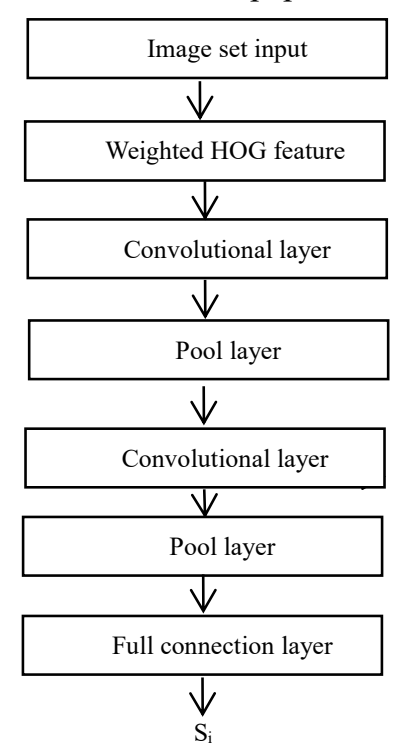

Figure 6. Convolutional neural network structure 
In the following, the convolutional layer, pooling layer and full connection layer of the convolution neural network will be mainly explained by formulas.

(1) Convolutional layer. The purpose of convolution operation is to extract different input features. The first convolutional layer may only extract some low-level features such as edges, lines and corners. The deeper network can extract more complex features iteratively from the low-level features. For example, the calculation formula [13-14] of the characteristic graph $j$ in the convolutional layer $l$ is:

$x_{j}^{l}=f\left(\sum_{i \in M i} x_{i}^{l-1} * W_{i j}^{l}+b_{j}^{l}\right)$

In the above formula, $X_{j}^{i}$ represents the output of the neuron $\mathrm{j}$ after convolution; the convolution kernel size is usually $5 * 5$ or $3 * 3$, and the function $f(x)$ represents a non-linear function as the activation function of this layer. $M i$ is the set of output characteristic graphs of the previous layer, ${ }^{*}$ represents convolution operation, and $w_{i j}^{l}$ and $b_{j}^{l}$ respectively represent convolution kernel and convolution layer offset.

(2) Pool layer. The pool layer is arranged between the two convolution layers and mainly performs downsampling operation on the characteristic map obtained by the convolution layer on the previous layer. This step can effectively reduce the matrix size, reduce the convolution feature dimension, and greatly reduce the number of training parameters to reduce the burden on the classifier, thus accelerating the calculation speed to a certain extent and preventing the data from over-fitting. Let the layer $l$ be a pool layer, and the calculation formula of a characteristic diagram of this layer is:

$x_{j}^{l}=\operatorname{down}\left(x_{j}^{l-1}\right)$

Where $\operatorname{down}(x)$ means the pooling operation.

(3) Full connection layer. The full connection layer mainly refits the features to reduce the loss of feature information. In fact, it mainly converts the convolution output two-dimensional feature map into one-dimensional feature vector. The calculation formula [13-14] is

$y=f(W * x+b)$

In the formula, $x$ represents the one-dimensional feature vector form of the two-dimensional feature map, and $w$ represents a matrix. Through the full connection process, any dimension feature map can be mapped into the specified dimension vector.

(4) Output layer. The output layer of convolutional neural network is used as a classifier, and the commonly used classifiers are sigmoid function, softmax function and centerloss function. In this paper, the output layer adopts softmax function, which has strong multi-category classification ability. As an image classification, softmax is a good choice. The formula of softmax function is

$S_{i}=\frac{e^{y_{j}}}{\sum_{j=1}^{C} e^{y_{j}}}$

In the above formula, $e^{y j}$ is the linear score function corresponding to the correct category, and $S_{i}$ is the Softmax output corresponding to the correct category.

\section{Simulation Experiment and Analysis}

In order to verify the feasibility and superiority of this algorithm, CMU PIE face database is used as the experimental data set. The experimental environment is python3.5.6+pytorch+tensorflow, InterCore I5 processor, 8G memory, Windows7 operating system. The experimental results are compared with $\mathrm{CNN}$, $\mathrm{HOG}+\mathrm{CNN}$, $\mathrm{HOG}+\mathrm{NMF}+\mathrm{CNN}$ methods respectively.

\subsection{Experimental data set}

CMU PIE face database has 68 objects, 112 images for each object and 7616 gray images. The image background 
is all single black, and the image size is $112 * 100$. The facial expressions and details of the face part have slight changes. The facial pose also has slight changes, and the illumination changes are obvious.

For CMU PIE face database, the image is divided into blocks with a size of $3 * 3$. HOG features are extracted from each image, multiplied by the weight coefficient of each image block, and the weighted HOG features are subjected to NMF dimensionality reduction to obtain the final block HOG features. The processed HOG feature is modeled, and the modeled image set is used as the input of CNN. Some face databases are shown in Figure 7:
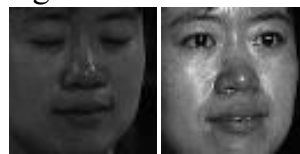
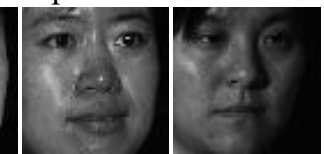

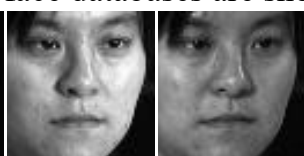

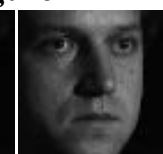

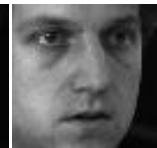

Figure 7. Some Face Images in CMU PIE Face Database

\subsection{Experimental parameter setting}

When extracting HOG features, the block size is $3 * 3$. The image set modeling is affected by $\mathrm{n}$, and $\mathrm{n}$ affects the number of image sets after modeling. In the experiment, $n=9,13,15,17$ and 20 are taken respectively. In the convolution neural network, bachsize is 8 , learning rate is 0.001 , and the number of convolution kernels is 6 and 16 in turn. The pooled size in the pool layer is $(2,2)$. When different values of $n$ are selected, CNN, HOG $+C N N$, $\mathrm{HOG}+\mathrm{NMF}+\mathrm{CNN}$ are compared with the algorithm in this paper. The experiment also tests the recognition rate of each algorithm when different training image sets are selected. The experimental results are shown in Tables 1-5. The change of recognition rate of each algorithm is shown in Figure 8.

\begin{tabular}{lllll}
\hline $\begin{array}{l}\text { Sample Number of Training Image } \\
\text { Sets }\end{array}$ & \multicolumn{2}{l}{10} & 9 & 10 \\
\hline CNN & 81.618 & 90.882 & 94.118 & 89.216 \\
HOG+CNN & 91.422 & 85.882 & 90.074 & 92.647 \\
HOG+NMF+CNN & 86.765 & 94.118 & 92.647 & 93.137 \\
Information entropy weighted & $\mathbf{8 8 . 3 7 8}$ & $\mathbf{9 5 . 4 2 1}$ & $\mathbf{9 3 . 1 2 5}$ & $\mathbf{9 4 . 0 8}$ \\
HOG+NMF+CNN (method in this & & & & \\
paper) & & & & \\
\hline
\end{tabular}

Table 1. Experimental results when $n=9$

\begin{tabular}{lllll}
\hline Number of training image sets & 4 & 5 & 6 & 7 \\
\hline CNN & 73.824 & 79.779 & 91.667 & 94.852 \\
HOG+CNN & 80.588 & 88.971 & 91.176 & 97.794 \\
HOG+NMF+CNN & 81.176 & 86.397 & 89.216 & 97.059 \\
Information entropy weighted & $\mathbf{8 3 . 6 2 3}$ & $\mathbf{9 1 . 4 2 9}$ & $\mathbf{9 0 . 3 7 4}$ & $\mathbf{9 8 . 1 1 4}$ \\
HOG+NMF+CNN (method in this & & & & \\
paper) & & & & \\
\hline
\end{tabular}

Table 2. Experimental results when $n=13$

\begin{tabular}{lllll}
\hline Number of training image sets & 3 & 4 & 5 & 6 \\
\hline CNN & 79.706 & 80.147 & 86.765 & 91.176 \\
HOG+CNN & 79.412 & 76.471 & 90.196 & 89.706 \\
HOG+NMF+CNN & 69.412 & 79.779 & 91.667 & 91.912 \\
Information entropy weighted & $\mathbf{8 3 . 7 7 9}$ & $\mathbf{8 6 . 3 9 7}$ & $\mathbf{9 0 . 5 5 9}$ & $\mathbf{9 4 . 8 5 2}$ \\
HOG+NMF+CNN (method in this & & & & \\
paper) & & & & \\
\hline
\end{tabular}

Table 3. Experimental results when $n=15$ 


\begin{tabular}{|c|c|c|c|c|}
\hline Number of training image sets & 2 & 3 & 4 & 5 \\
\hline $\mathrm{CNN}$ & 60.294 & 72.794 & 84.804 & 83.824 \\
\hline $\mathrm{HOG}+\mathrm{CNN}$ & 67.941 & 76.471 & 84.412 & 87.50 \\
\hline $\mathrm{HOG}+\mathrm{NMF}+\mathrm{CNN}$ & 56.471 & 82.721 & 85.784 & 91.176 \\
\hline $\begin{array}{l}\text { Information entropy weighted } \\
\mathrm{HOG}+\mathrm{NMF}+\mathrm{CNN} \text { (method in this } \\
\text { paper) }\end{array}$ & 69.485 & 79.623 & 88.216 & 93.059 \\
\hline \multicolumn{5}{|c|}{ Table 4. Eexperimental results when $n=17$} \\
\hline Number of training image sets & 1 & 2 & 3 & 4 \\
\hline $\mathrm{CNN}$ & 35 & 55.882 & 78.922 & 85.294 \\
\hline $\mathrm{HOG}+\mathrm{CNN}$ & 45.294 & 72.794 & 81.863 & 83.088 \\
\hline $\mathrm{HOG}+\mathrm{NMF}+\mathrm{CNN}$ & 34.412 & 69.324 & 82.294 & 86.765 \\
\hline $\begin{array}{l}\text { Information entropy weighted } \\
\mathrm{HOG}+\mathrm{NMF}+\mathrm{CNN} \text { (method in this } \\
\text { paper) }\end{array}$ & 40.059 & 65.294 & 84.412 & 89.824 \\
\hline
\end{tabular}

Table 5. Experimental Results when $n=20$

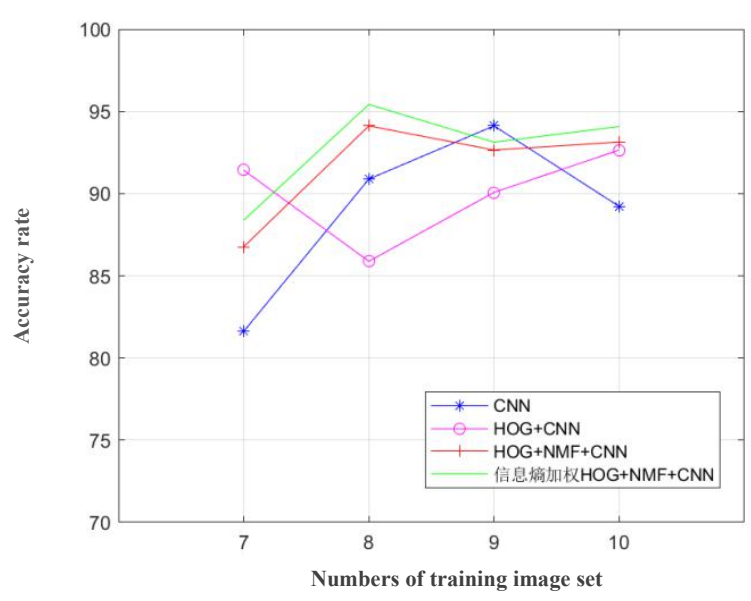

(a) face image set recognition rate in $n=9$

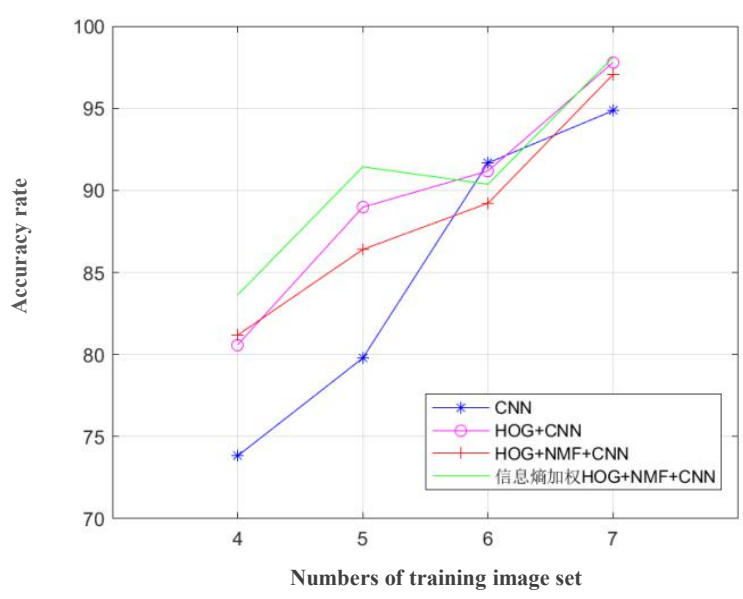

(b) face image set recognition rate in $n=13$ 


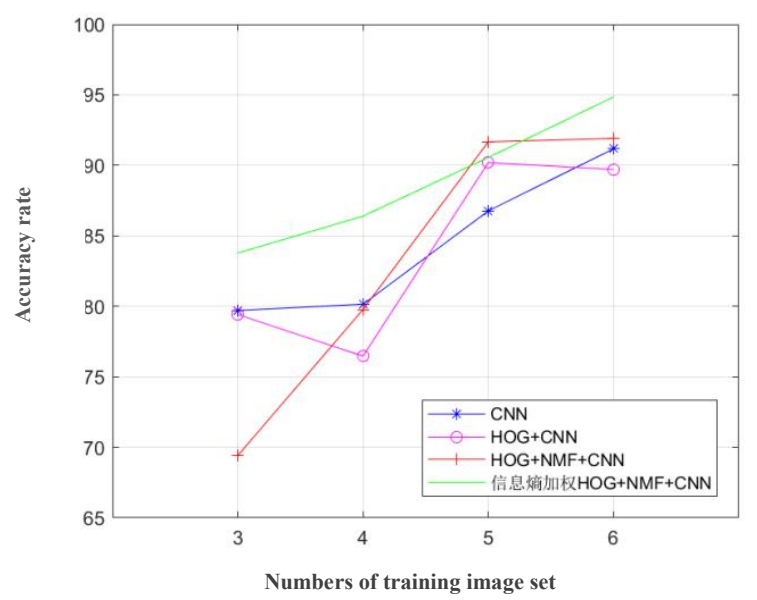

(c) face image set recognition rate in $n=15$

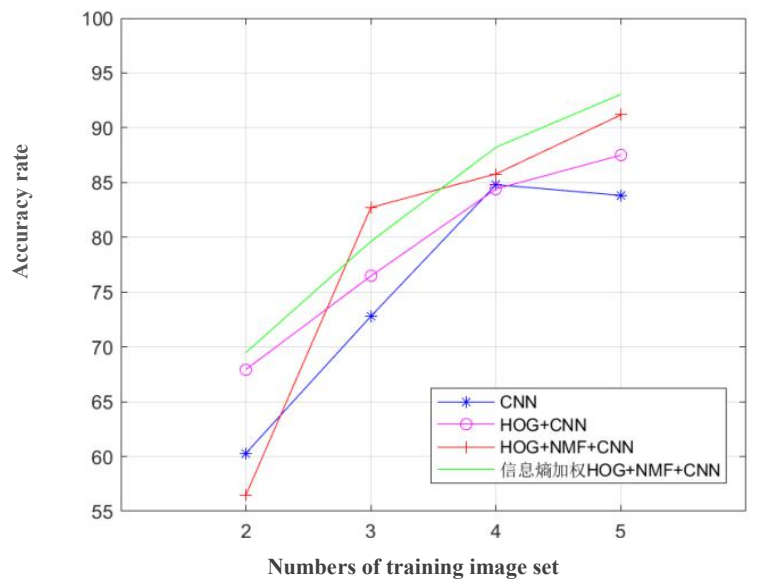

(d) face image set recognition rate in $n=17$

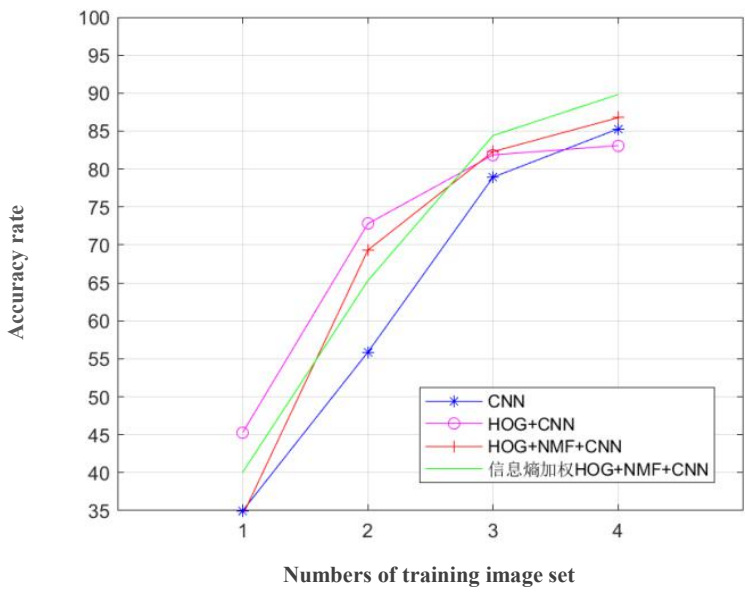

(e) face image set recognition rate in $n=20$

Figure 8. Recognition rate of face image sets with different values of $n$

As can be seen from figure 8, under the conditions of $n=9, n=13, n=15, n=17$ and $n=20$, after modeling the CMU_PIE image database, there are 13, 9, 8, 7 and 6 image set samples corresponding to each person. Therefore, according to the different values of $n$, different numbers of training image sets and test image sets are selected in the experiment. The experimental results show that compared with the previous methods, the recognition accuracy of the proposed method in face image set recognition has been improved to a certain extent. This is due to the modeling of image set recognition, which preserves the details of each person and makes face recognition more accurate. Moreover, 
the combination of weighted HOG features and NMF dimensionality reduction proposed in this paper makes the recognition rate significantly higher. The main reason is that weighted HOG feature extraction makes the details of the face more prominent according to the different weighted values of the information contained in each block of the face, such as the nose and eyes of the face. After NMF dimensionality reduction, the data dimension is reduced. At the same time, the positive vectors useful for face recognition are saved, and then the NMF dimensionality reduction image is modeled to save the details of each person's various angles as much as possible, thus improving the recognition rate of face image sets.

\section{Conclusion}

In this paper, an improved HOG feature extraction method is proposed, and the NMF method is used to reduce the dimension. After modeling the image set, the new method of face image set recognition is combined with CNN. HOG features can fully describe the local features of the face, effectively avoid the influence of unfavorable factors such as illumination, and then reduce the dimension of the face features to obtain the main face features. The image set modeling retains the face details. The face features obtained after modeling are combined with $\mathrm{CNN}$, which can further extract the face feature information. The experimental results of CMU_PIE face database show that the proposed method can effectively improve the recognition rate of face image sets. In the aspect of image set modeling, the arithmetic mean method adopted in this paper does not take the relationship between different gestures, expressions and other details of the same person into account, which needs further discussion.

\section{References}

1. He C. A Survey of Face Recognition Technology [J]. Intelligent Computers and Applications 2016; 6(1): 112-114.

2. Dong XW, Yao SM, Wang YW, et al. Multi-manifold discrimination learning algorithm based on virtual sample image set[J]. Application Research of Computers 2018; 35(06): 1872-1878.

3. Zhang WK, Sun H, Sun X, Wang HQ. Visual Summary of Image Set Based on MFF-GAN [J]. Computer Engineering 2019; 45(02): 202-206.

4. Ren $\mathrm{ZW}, \mathrm{Wu} \mathrm{MN}$. Image set classification algorithm based on entropy self-weighted joint regularization nearest

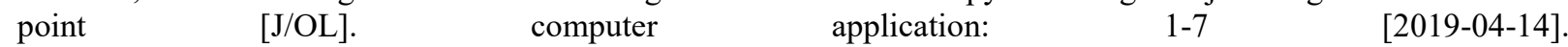
http://KNS.cnki.net/kcms/detail/51.1307.TP.20190508.1047.002.html)

5. Dalal N, Triggs B. Histograms of Oriented Gradients for Human Detection [C]. Computer Vision and Pattern Recognition 2005. Computer Society Conference on IEEE 2005: 886-893.

6. Xie Z, Jiang P, Zhang S. Fusion of LBP and HOG using multiplekernel learning for infrared face recognition [C]. IEEE/ACIS International Conference on Computer and Information Science 2017: 81-84.

7. Bhele SG, Mankar VH. Recognition of faces using discriminative features of LBP and HOG descriptor in varying environment [C]. International Conference on Computational Intelligence and Communication Networks 2016: 426-432.

8. Ghorbani M, Targhi AT, Dehshibi MM. HOG and LBP: Towards a robust face recognition system [C]. Tenth International Conference on Digital Information Management 2016: 138-141.

9. Sun R, Chen J, Gao W. A Fast Pedestrian Detection Method Based on Significance Detection and HOG-NMF Features [J]. Journal of Electronics \& Information Technology 2013; 35(08): 1921-1926.

10. Lin KZ, Zhang YM, Li YT. Research on HOG feature extraction algorithm based on information entropy weighting $[\mathrm{J} / \mathrm{OL}]$. computer engineering and applications: $1-10$ [2019-05-06]. http://kns.cnki.net/kcms/detail/11.2127.tp.20190411.1719.016.html.

11. Wang HB, Chen YX, Li YQ. Face recognition method based on principal component analysis and Softmax regression model [J]. Journal of Hefei University of Technology (Natural Science) 2015; 38(06): 759-763.

12. Zhang YM. Research on Face Recognition Algorithm Based on HOG and Gabor Features [D]. Harbin University of Science and Technology 2019.

13. Lee H, Yoo J, Choi S. Semi-supervised nonnegative matrix factorization [J]. IEEE Signal Processing Letters 2010; 17(1): 4-7.

14. Syafeeza AR, Khalil-Hani M, Liew SS, et al. Convolutional neural network for face recognition with pose and illumination variation [J]. International Journal of Computational Intelligerce \& Applications 2015; 14(3): 1-18.

15. Hou XP, Gao Y. Application of Convolutional Neural Network CNN Algorithm in Text Classification [J]. Technology and Innovation 2019(04): 158-159. 